\title{
A DOUBLET C0 IR SOLUTION USING EXISTING MAGNETS
}

JOHN A. JOHNSTONE

Fermilab, Batavia, IL 60510-0500

This note explores, in a very limited way, some of the possibilities \& difficulties encountered in creating collisions at $\mathrm{C} 0$ solely using magnets looted from the existing IR's at B0 \& D0. In the strawman model considered here a standard Collins straight section is installed at D0, while all the useful D0 IR magnets are moved to $\mathrm{C} 0$. There is no obvious reason from either an optics or beamseparation viewpoint to prefer the demise of D0 over CDF, or vice-versa, so the model choice is fairly arbitrary. Apart from modifications to the final-focus optics, the magnets appear at $\mathrm{C} 0$ locations just as they are currently installed at D0.

\section{PHYSICAL LAYOUT}

The magnet layout and power circuits of the model C0 IR are illustrated in Figure 1, with the bizarre Tevatron magnet numbering scheme of the CDF/D0 IR's being retained. In Run II CDF \& D0 use 4 additional trim quads to those indicated below. These are independently-powered regular tune-quad spools which, in the picture below, would correspond to QT8's at the B45/C15 locations, and QTB \& QTA spools at the B39 \& B42 sites. Since in the C0 colliding mode envisioned here CDF doesn't experience collisions concurrently with $\mathrm{C} 0$, the additional fine-tuning provided by 4 more trims is unwarranted.

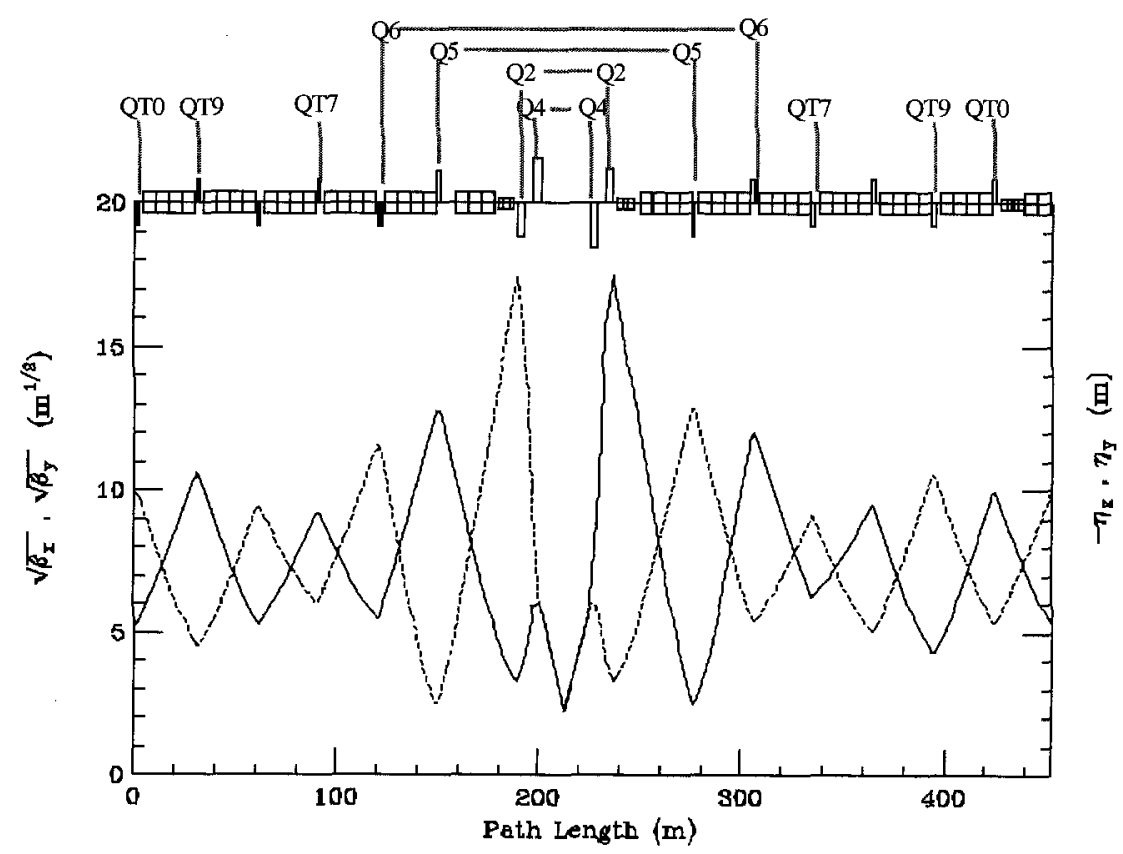

Figure 1. Series \& independently-powered circuits of the CO Interaction Region. 


\section{OPTICS}

It does not appear possible to create triplet final-focus optics at $\mathrm{C} 0$ with existing Tevatron IR magnets. Currently at CDF/D0 the IP to triplet distance is $\pm 7.363 \mathrm{~m}$. The BTeV detector requires that this space be expanded to at least $\pm 12.192 \mathrm{~m}$. By eliminating the $\mathrm{Q} 1$ magnet plus its accompanying TSP spool, and replacing them with a single short (49.91") TSS spool, an extra $2.008276 \mathrm{~m}$ of space can be created. It's difficult to imagine, though, how an additional $2.8209 \mathrm{~m}$ of space could be generated, producing sufficient room for both a triplet plus the detector.

\begin{tabular}{|c|c|c|c|}
\hline Quad Location & $\begin{array}{c}\text { Magnetic Length } \\
\text { (in ) }\end{array}$ & $\begin{array}{c}\text { Max. Gradient } \\
\text { ( T/m })\end{array}$ & $\begin{array}{c}\text { Max. Current } \\
\text { ( A ) }\end{array}$ \\
\hline Q4/Q2 & 132 & 145 & 5000 \\
\hline$[\mathrm{Q} 3]$ & {$[232]$} & {$[145]$} & {$[5000]$} \\
\hline$[\mathrm{Q} 1]$ & {$[55.19]$} & {$[145]$} & {$[5000]$} \\
\hline Q5 & 55.19 & 145 & 5000 \\
\hline Q6 & 23.875 & 145 & 5000 \\
\hline QT7/QT9/QT0 & 25 & 58 & 1000 \\
\hline
\end{tabular}

Table 1. Magnet parameters of the available high-gradient D0 quadrupoles. (Shaded entries are not used in the present CO design).

While doublets do have the advantage over triplets of requiring less space \& generally lower gradients, they have the glaring drawback that $\beta \max$ is $3-4$ times larger than it would be in a triplet for the same $\beta^{*}$. Doublet optics at C0 can be created with the Q4 \& Q2 quadrupoles or, if the Q1+TSP combination is removed as described above, with the Q3 \& Q2 quadrupoles.

In Run II the Tevatron operates with tunes near the half-integer; at $\left(\mu_{\mathrm{X}}, \mu_{\mathrm{y}}\right)=(20.585,20.575)$. There are two simple approaches that maintain the nominal operating point. The obvious solution is to leave CDF tuned to its Collider mode injection lattice. By simply moving the other IR from D0 to $\mathrm{C} 0$ the overall machine tune is unaffected. Another option is to re-tune CDF to the Fixed Target lattice, in this case the B0 insert becomes a "Collins-like" straight. The additional half-integer of tune is then generated by the regular tune-quad strings and becomes distributed uniformly around the ring. The CDF Lattice functions \& gradients for these 2 options are provided in Appendix A.

The first option has the advantage that B0 can be easily re-tuned for collisions when $\mathrm{C} 0$ is not in use. However, it will be seen later that this solution creates significant difficulties for maintaining adequate beam separation around the ring. The second approach - re-tuning B0 to Fixed Target optics - is greatly preferred from the standpoint of beam separation. Unfortunately, the drawback in this case is that a shift or so of labor is required to physically switch powering at B0 between Fixed Target \& Collider optics. 
C0 injection \& collision optics \& gradients for this model are shown in Figures 2 \& 3 and Tables 2 $\& 3$, respectively. The tabulated results reflect the assumption of Fixed Target optics at CDF, but the qualitative differences compared with CDF Collider optics are not significant. Variation of the main quad gradients through the $\beta^{*}=7.00 \rightarrow 1.50 \mathrm{~m}$ squeeze are illustrated in Figure 4 .

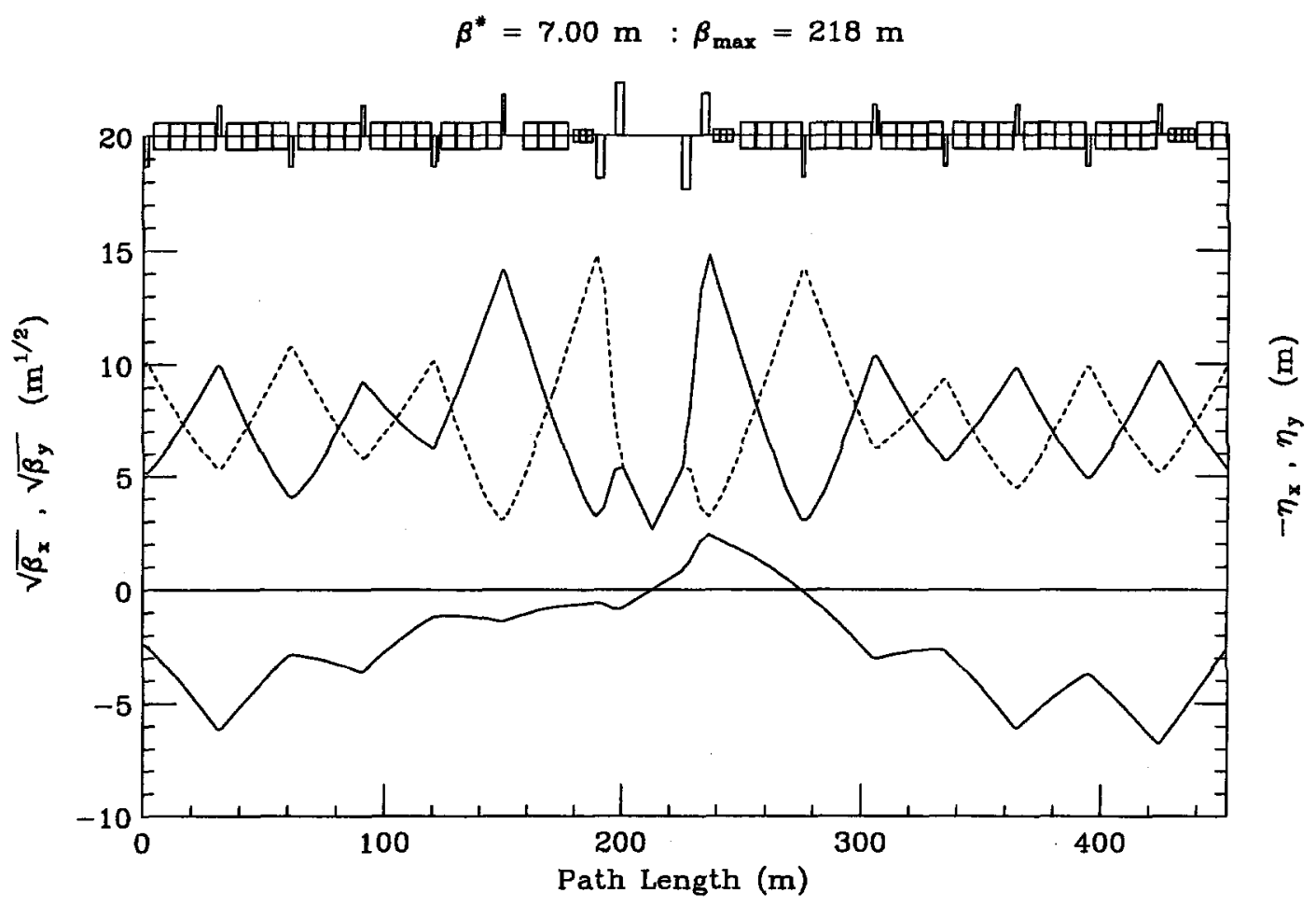

Figure 2. C0 injection optics.

\begin{tabular}{|c|c|c|}
\hline Quad \# & \multicolumn{2}{|c|}{$\begin{array}{ll}\text { Co Gradients @ } \beta^{*}= & 7.00 \mathrm{~m} \\
\left.\operatorname{up}_{(\mathrm{T} / \mathrm{mn}}\right) & (\mathrm{T} / \mathrm{m}) \\
\end{array}$} \\
\hline Q4 & 133.4729 & -133.4729 \\
\hline $\mathrm{Q} 2$ & -106.0786 & 106.0786 \\
\hline Q5 & 102.4556 & -102.4556 \\
\hline Q6 & -60.2982 & 60.2982 \\
\hline QT6 & 0.4753 & 4) \\
\hline QT7 & 5.3936 & 3.0547 \\
\hline QT9 & 34.0098 & -24.2252 \\
\hline QT0 & 0.6680 & 13.6446 \\
\hline
\end{tabular}

Table 2. Injection gradients. 


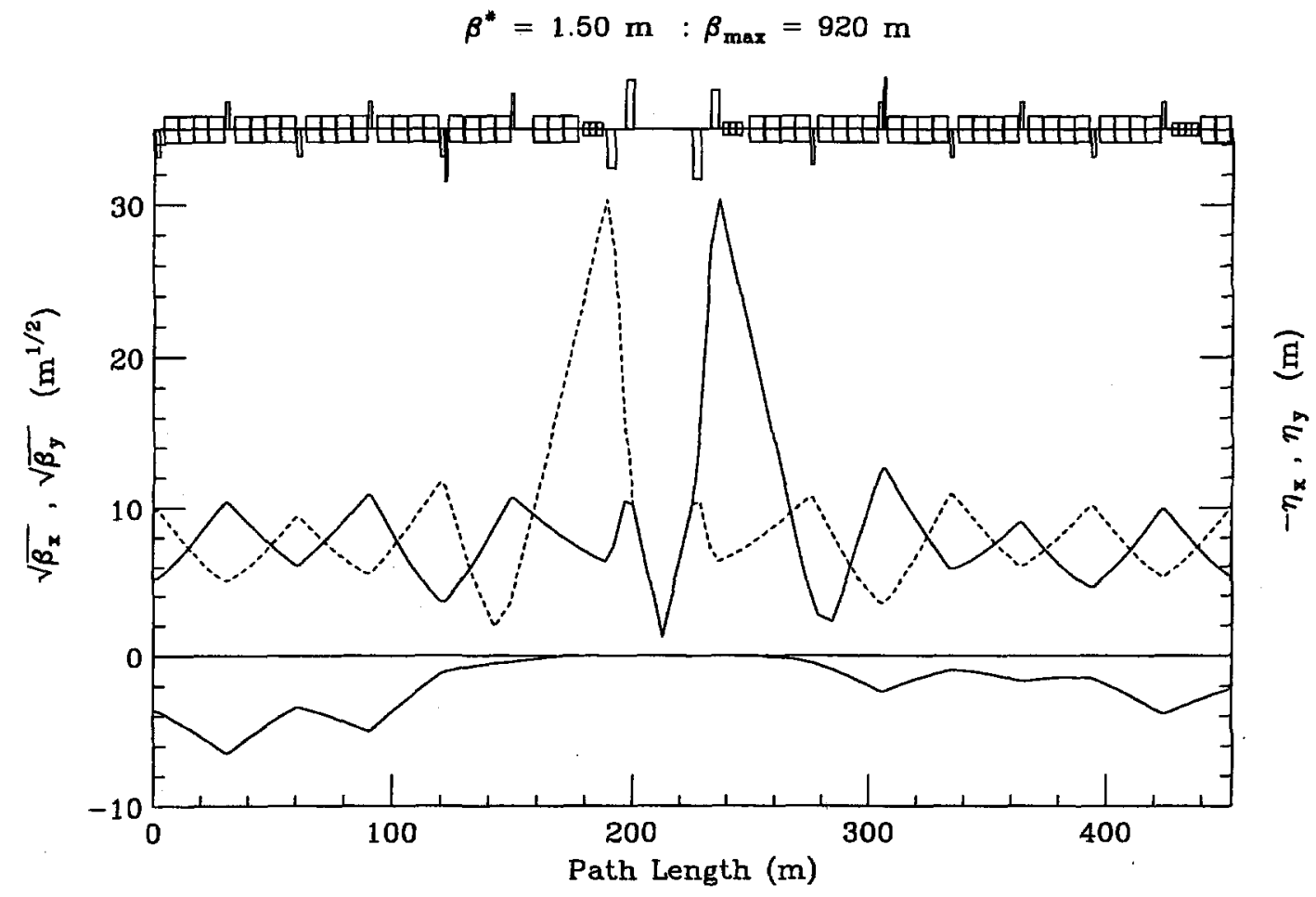

Figure 3. Collision optics

\begin{tabular}{||c||c|c||}
\hline \hline Quad \# & $\begin{array}{c}\text { C0 Collision Gradients @ } \beta^{*}=1.50 \mathrm{~m} \\
\text { up } \\
\text { down } \\
(\mathrm{T} / \mathrm{m} / \mathrm{m})\end{array}$ \\
\hline \hline Q4 & 137.8122 & -137.8122 \\
\hline Q2 & -108.0504 & 108.0504 \\
\hline Q5 & 96.7136 & -96.7136 \\
\hline Q6 & -144.0000 & 144.0000 \\
\hline QT6 & -7.0804 & \\
\hline QT7 & 33.9576 & -39.4609 \\
\hline QT9 & -8.4904 & 8.7652 \\
\hline QT0 & -7.2144 & 11.9285 \\
\hline
\end{tabular}

Table 3. Collision gradients. 
High-Field Quad Gradient Variations with $\beta^{*}$

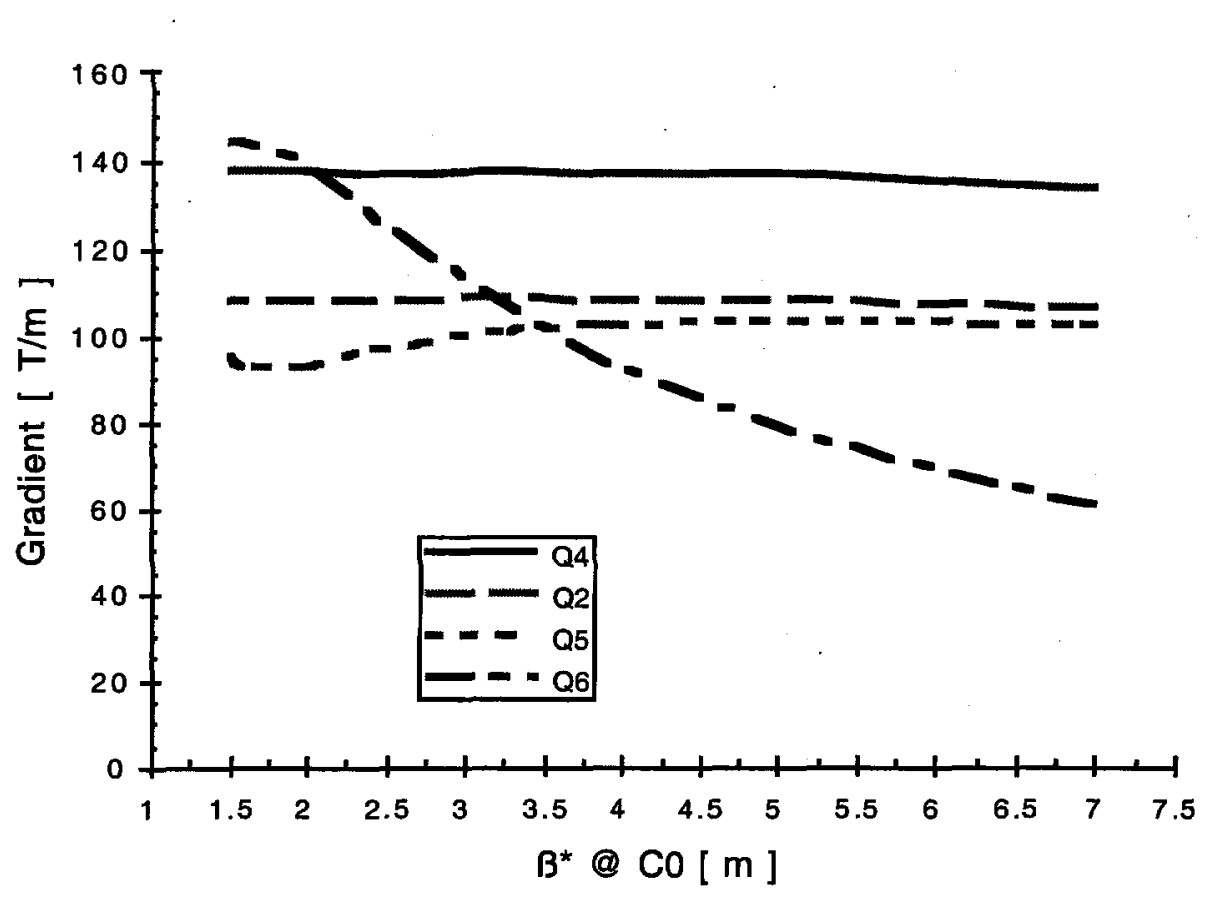

Figure 4. Gradients of the main quadrupoles through the $\beta^{*}=7.00 \rightarrow 1.50 \mathrm{~m}$ squeeze.

At injection, $\beta^{*}$ can be increased from the $7.00 \mathrm{~m}$ shown here, but there is no advantage to this. With $\beta^{*}=7.00, \beta \max =220 \mathrm{~m}$ at both the doublets $\&$ the $\mathrm{B} 48 / \mathrm{C} 12$ locations (this is already less than the $\sim 250 \mathrm{~m}$ of the $A 0$ high- $\beta$ insert \& the standard CDF/D0 injection lattices). Increasing $\beta^{*}$ further simply drives up $\beta$ max at B48 \& C12. Dispersion at the IP is zero but $\eta^{\prime *} \neq 0$. For such large beams there are no compelling reasons for having the slope of $\eta$ also tuned to zero.

For collisions, $\beta^{*}=1.50 \mathrm{~m}$ and the optics are tuned to make both $\eta^{*} \& \eta^{\prime *}=0$. Again, for such large beams no solid argument can be made for insisting that $\eta^{\prime *}=0$ at the IP, other than it soothes the author's aesthetic sensibilities. The minimum attainable value of $\beta^{*}$ is determined by the maximum gradient limit reached by the $\mathrm{Q} 6$ quadrupoles. More extensive exploration of quadrupole positions, allocation of quads to different arc locations, etc., might allow $\beta^{*}$ to be lowered further. However, any gains will ultimately be limited by the beam size in the doublets - a $\beta \max =920 \mathrm{~m}$ for $\beta^{*}=1.50 \mathrm{~m}$ implies that $\beta \max$ will become $\sim 1050 \mathrm{~m}$ for $\beta^{*} \sim 1.3 \mathrm{~m}$, which is then comparable to $\beta \max$ in the current CDF/D0 triplets with $\beta^{*}=0.35 \mathrm{~m}$ 


\section{BEAM SEPARATION}

In Run IIb bunch spacing in the Tevatron will be reduced from $396 \rightarrow 132$ nsec to decrease the number of interactions per crossing at the detectors. It is assumed here that this operational mode will continue during the $\mathrm{BTeV}$ era. With the first parasitic crossings then occurring just $19.76 \mathrm{~m}$ from the IP a crossing angle becomes unavoidable to obtain separated beams at these points.

There are at least 2 consequences of introducing a crossing angle. First, luminosity is reduced due to the decreased overlap of the beams at the IP. This is not expected to be a huge effect in the current model, however, because of the sizes of the colliding beams. The greater concern is that a crossing angle produces separated beams in the final-focus doublets - precisely where the beam already reaches its ring-wide maximum. The impact of high-order multipoles on the beams traveling off-axis through the quadrupoles is not known with any degree of confidence - the Tevatron has never been operated with crossing angles before.

Creating adequately separated beams with doublet optics is more difficult than with triplet finalfocusing. The displacement at some point in the arcs due to a kick $\theta$ from the separators is:

$$
\Delta x(y)=\sqrt{\beta_{\text {sep }} \cdot \beta_{x(y)}} \cdot \sin \left(\Delta \Psi_{x(y)}\right) \cdot \theta_{\text {sep }}
$$

At the $\mathrm{CDF} / \mathrm{D} 0$ separators the beta function in one plane relative to the other is:

$$
\text { Triplets: } \quad \sqrt{\beta_{>} / \beta_{<}} \approx 2
$$

So, at CDF \& D0, to produce a reasonably round helix in the arcs, effectively equal kicks are achieved by having 2 separators in the plane with the smaller $\beta$ and 1 separator in the other plane.

Three separators is not the right number with a doublet final-focus. A quick inspection of Figure 3 reveals that the ratio of $\beta$-functions at the separators is such that:

Doublets :

$$
\sqrt{\beta_{>} / \beta_{<}} \approx 31 / 2 \rightarrow 4
$$

For doublets then the appropriate number of separators is not 3 , but more like 4 or 5 !

In the present model it didn't seem possible to generate enough room for an additional separator at the B49 \& C11 locations consistent with quad gradient limitations. The solution decided upon here is to roll the separator in the plane of larger $\beta$ through $45^{\circ}$. This effectively increases the separator number in one plane from 2 to 2.71 while reducing it in the other from 1 to 0.71 - thereby creating an effective kick ratio of $\approx 3.83: 1$; in keeping with the desire for round helices. 
In the current Tevatron Collider lattice separator locations are well suited for creating collisions at CDF \& D0, while keeping the beams separated everywhere else in the ring. There are very nearly multiples of $\sim 180^{\circ}$ separating all of the A49, B11, C49, and D11 separators. Also, the "17" straights are roughly multiples of $\sim 90^{\circ}$ from the IP's and the A49/B11, C49/D11 separators. The picture is not quite this clean in detail, of course but, nonetheless, it is pretty straightforward to construct appropriate three-bumps to control both beam position \& angle at the IP's with good separation elsewhere.

With one IR moved from D0 to C0 the phase relation between the separators \& IP deteriorates considerably. In the vertical plane, in particular, there are now roughly odd-multiples of $45^{\circ}$ from the separators to the IP all through the A \& B sextants. It is still possible to create the 3-bumps required to fix position \& angle at the IP, but only by powering many of the vertical separators at their maximum gradients \& installing separators in some unorthodox locations.

One possible separator solution is demonstrated in Figure $5 \&$ Table 4. Half-crossing angles of $\left(\mathrm{x}^{\prime *}, \mathrm{y}^{\prime *}\right)=(-130,-130) \mu \mathrm{rad}$ give $8 \sigma$ of separation at the 1st parasitic crossing, but just $5 \sigma$ at the 5th \& 6th crossings, for $20 \pi \mu \mathrm{m}$ (95\%, normalized) beams. It's clear that separation falls apart badly through A-sector, even with 5 new separators jammed into the A0 straight amidst the 5 proton abort kickers, 5 pbar abort kickers, collimator, and 2 abort blocks. Note also that a total of 31 separator modules are needed for this solution -9 more than the Run I inventory.

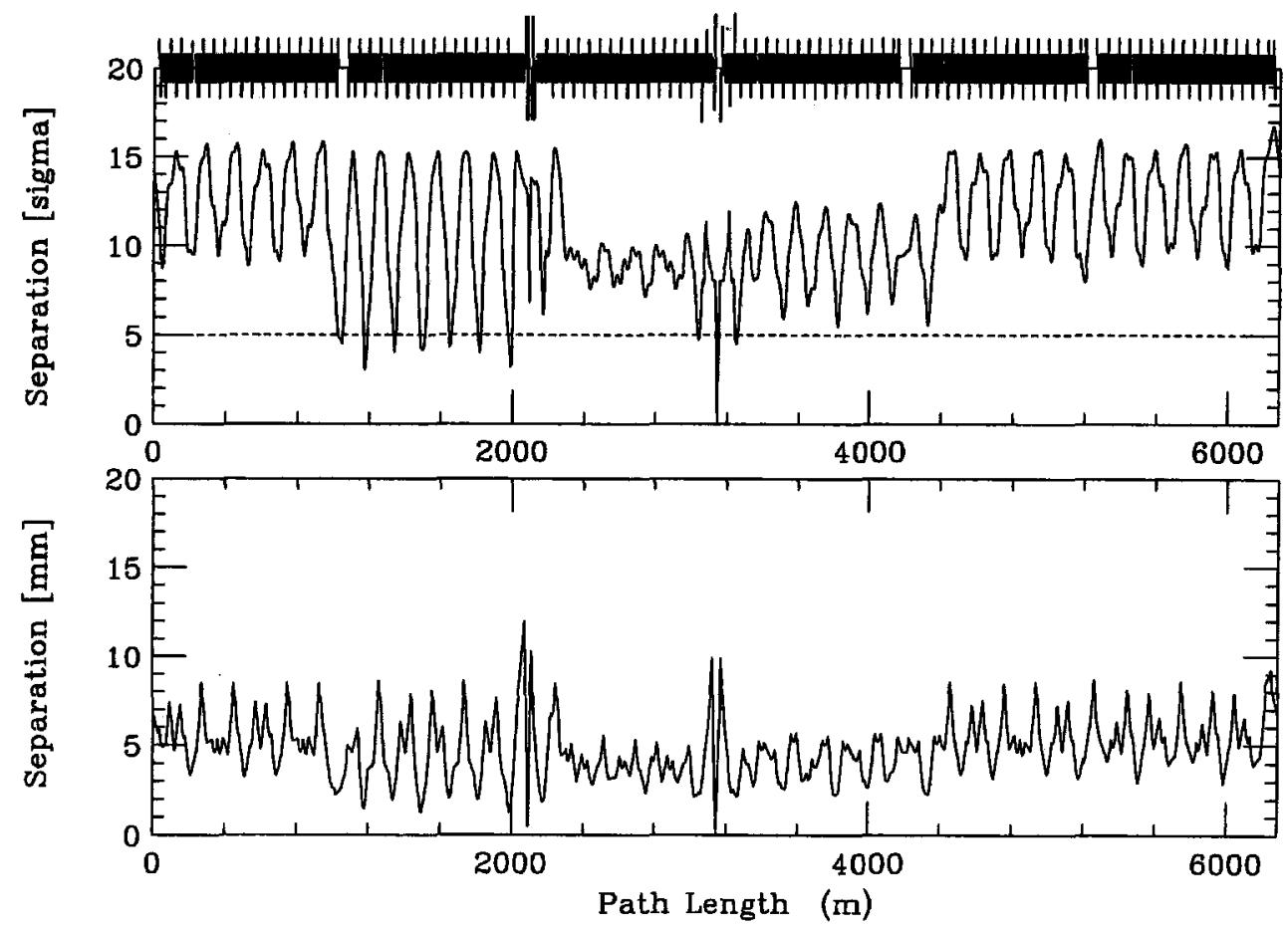

Figure 5. Beam separation with CDF optics tuned to the Collider Injection lattice. 


\begin{tabular}{||c|c|c|c|c|c||}
\hline \multicolumn{5}{|c|}{$\begin{array}{c}\text { Separator Gradients } \\
\text { (MV / m ) }\end{array}$} \\
\hline & & & A0 & 5 & 3.35591 \\
\hline & & & A17 & 2 & -1.22490 \\
\hline A49 & 1 & -3.47656 & A49 & 2 & -4.00000 \\
\hline B11 & 2 & 3.47656 & B11 & 1 & 4.00000 \\
\hline B17 & 4 & -2.51233 & & & \\
\hline & & & B 48 & 1 & -4.00000 \\
\hline B49 & 2 & 4.00000 & B49 & 1 & 4.00000 \\
\hline C11 & 1 & -4.00000 & C11 & 2 & -4.00000 \\
\hline & & & C17 & 4 & 2.53886 \\
\hline D17 & 3 & -3.69073 & & & \\
\hline
\end{tabular}

Table 4. Separator gradients with CDF optics tuned to the Collider Injection lattice.

A preferred separation scheme is illustrated in Figure 6 \& Table 5. In this case CDF is re-tuned to the Fixed Target lattice \& the tune-quad strings are used to restore the nominal tune operating point. The required gradients of QFA4 $=2.450 \mathrm{~T} / \mathrm{m} \& \mathrm{QDD} 1=-4.457 \mathrm{~T} / \mathrm{m}$ are comfortably within the $\sim 9 \mathrm{~T} / \mathrm{m}$ range of these spool magnets. With the additional half-integer of tune distributed around the ring, rather than localized at CDF, separator solutions become much easier to find.

The solution shown below again has half-crossing angles of $\left(x^{\prime *}, y^{\prime *}\right)=(-130,-130) \mu$ rad to create $5 \sigma$ separation at the 5th $\& 6$ th crossings. Elsewhere in the ring beam separation doesn't drop below about $7 \sigma$, with the average being $\sim 10 \sigma$. The vertical solution includes an extra 3-bump from C17-D48-E17 solely to help smooth the orbit through C-sector. This refinement might not be strictly necessary since without the additional smoothing beam separation still remains above $\sim 6 \sigma$ in this region. Even with the extra D48 \& E17 separators this solution uses fewer separator modules than were available during Run I. 


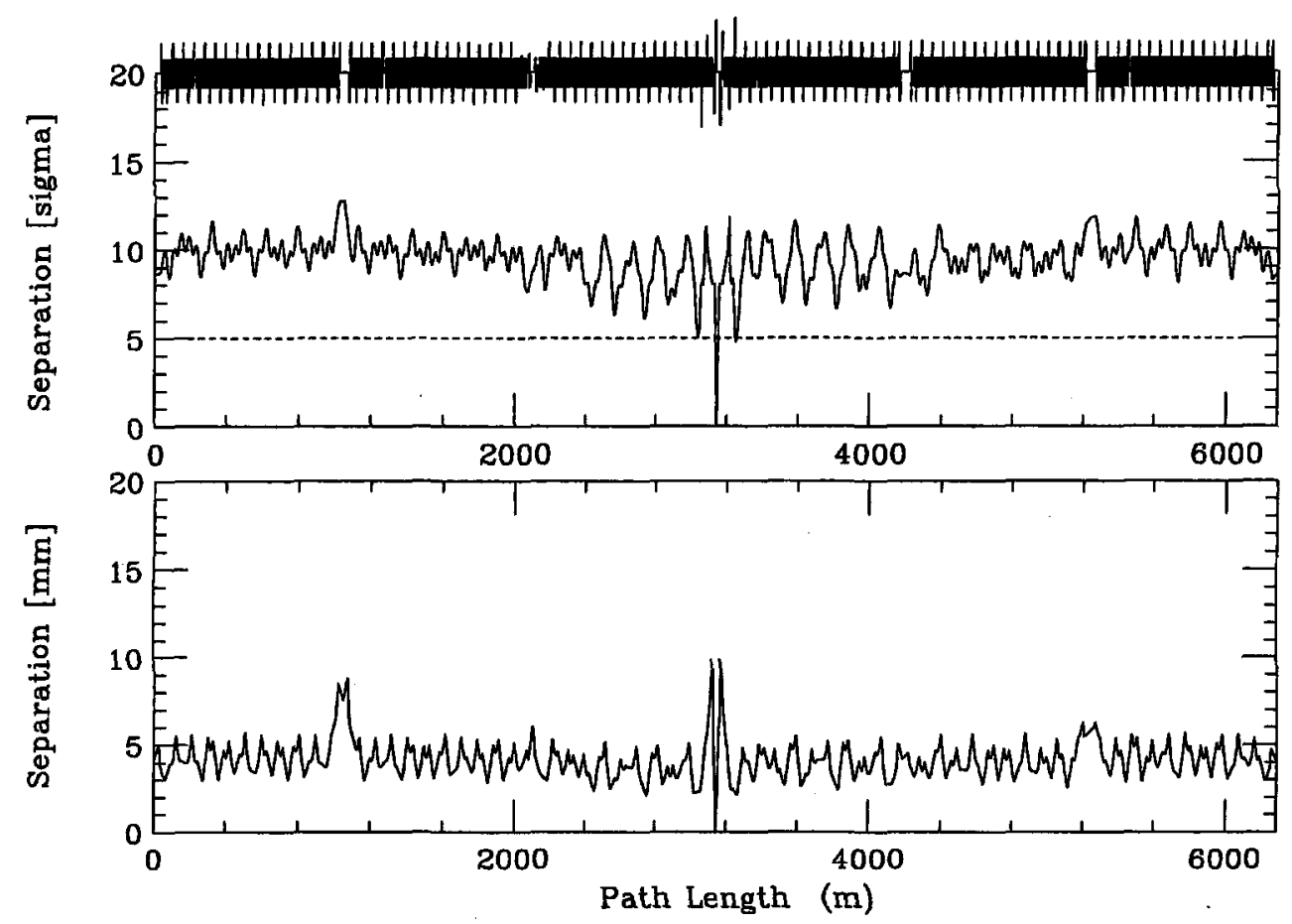

Figure 6. Beam separation with CDF optics tuned to the Fixed Target lattice.

\begin{tabular}{||c|c|c|c|c|c||}
\hline \multicolumn{7}{|c||}{$\begin{array}{c}\text { Separator Gradients } \\
\text { (MV/m ) }\end{array}$} \\
\hline & & & A17 & 2 & 0.26792 \\
\hline B17 & 4 & -1.47608 & & & \\
\hline B49 & 2 & 4.00000 & B49 & 1 & 4.00000 \\
\hline C11 & 1 & -4.00000 & C11 & 2 & -4.00000 \\
\hline & & & C17 & 4 & 2.01205 \\
\hline D17 & 2 & -1.79396 & & & \\
\hline & & & D48 & 1 & 2.24464 \\
\hline & & & E17 & 2 & -3.30601 \\
\hline
\end{tabular}

Table 5. Separator gradients with CDF optics tuned to the Fixed Target lattice. 


\section{PERFORMANCE SUMMARY}

The expression for luminosity can be written in terms of a few basic machine \& IP parameters plus a form factor which contains details of the beams at the IP:

$$
L=\frac{f \cdot B \cdot N_{1} N_{2}}{4 \pi \cdot \beta^{*} \cdot \varepsilon_{N_{95}} / 6(\beta \gamma)_{R}} \cdot F\left(\beta^{*}, \theta_{1 / 2}\right)
$$

Here, $\mathrm{f}$ is the revolution frequency, $\mathrm{N}_{1} \& \mathrm{~N}_{2}$ are the number of protons \& pbars per bunch, $\mathrm{B}$ is the number of bunches, $\varepsilon_{\mathrm{N} 95}$ is the $95 \%$ (normalized) emittance ${ }^{1}$, and $(\beta \gamma)_{\mathrm{R}} \equiv \mathrm{E} / \mathrm{m}$. The form factor $\mathrm{F} \leq 1$ contains both the "hourglass" \& crossing angle effects 2 :

$$
F\left(\beta^{*}, \theta_{1 / 2}\right) \equiv \frac{2}{\sqrt{\pi}} \cdot z \cdot e^{-z^{2} \theta^{2}} \cdot \int_{0}^{\infty} \frac{d \mu}{\left(1+\mu^{2}\right)} \cdot \exp \left\{-z^{2} \mu^{2}+\frac{z^{2} \theta^{2}}{\left(1+\mu^{2}\right)}\right\}
$$

where $\theta$ and $\mathrm{z}$ are defined in terms of $\beta^{*}$ and the total half-crossing angle $\theta_{1 / 2}$ as:

$$
\theta=\theta_{1 / 2} \cdot \frac{\sigma_{1}}{\sigma_{t}^{*}}, \text { and } ; \quad z=\beta^{*} / \sigma_{1}
$$

with $\sigma_{1}$ and $\sigma_{t}^{*}$ being the rms longitudinal \& transverse beam sizes, respectively. From these definitions the luminosity calculated for $\mathrm{C} 0$ is given in Table 4 for typical Run $\mathrm{Ilb}$ parameters.

\begin{tabular}{|l|c|r|}
\hline Protons/bunch & 27 & $10^{10}$ \\
\hline Proton Emittance & $20 \pi$ & $\mu \mathrm{m}$ \\
\hline Antiprotons/bunch & 10 & $10^{10}$ \\
\hline Antiproton Emittance & $20 \pi$ & $\mu \mathrm{m}$ \\
\hline Bunch Length & 0.37 & $\mathrm{~m}$ \\
\hline Number of p \& pbar Bunches & 108 & $\mathrm{nsec}$ \\
\hline Bunch Spacing & 132 & $\mathrm{kHz}$ \\
\hline Revolution Frequency & 47.71 & $\mathrm{~m}$ \\
\hline$\beta^{*}$ & 1.50 & $\mathrm{GeV}$ \\
\hline Energy & 1000 & $\mu \mathrm{rad}$ \\
\hline Total Half-Crossing Angle & 185 & 1.6 \\
\hline Initial Luminosity & & $10^{32} \mathrm{~cm}^{-2} \mathrm{~s}^{-1}$ \\
\hline
\end{tabular}

Table 4. Run IIb performance parameters for the $\mathrm{C} 0$ doublet IR.

\footnotetext{
1 For simplicity B \& $\varepsilon_{N 95}$ are assumed to be the same for protons \& pbars.
}

2 This expression for $F$ is valid for $\left|x^{\prime} *\right|=\left|y^{\prime} *\right|$. 


\section{DIRECTIONS FOR FUTURE STUDY}

The model analysis presented here represents only the most cursory examination of the issues associated with developing a functional doublet final-focus IR at C0. However, although individual doublet designs are sure to differ in detail, their fundamental optical properties will almost certainly be similar.

The most significant demonstration of the current study is probably that the major obstacles to a satisfactory solution are not connected directly to optics issues, but rather arise from difficulties encountered in obtaining adequate beam position \& angle control throughout the ring. At the heart of this problem is the large mis-match previously noted between the ratio of $\beta$-functions at the separators immediately outboard of the IP \& the number of separators available to initiate the helix. The solution arrived at in this note was to roll one module each side of the IP by $45^{\circ}$ as compensation. This somewhat desperate remedy, however, is extremely odious. Not only does the rotation impact the separator aperture, but also couples the solutions between the horizontal \& vertical planes.

Subsequent studies should thoroughly explore the options for creating an IR optics solution that generates enough warm space to install a 4th separator each side of the IP — hopefully eliminating the need for separator rotations. This might be achieved with a more imaginative configuration of arc matching quadrupoles, for instance.

Failing this, or perhaps in addition, the implications of relaxing the $\alpha^{*} \equiv 0$ constraint should be fully examined. While $\alpha_{\mathrm{x}}{ }^{*}=-\alpha_{\mathrm{y}}{ }^{*} \neq 0$ will reduce luminosity for a fixed $\beta^{*}$, the impact is moderated to some extent because, in moving away from the IP, the beam is growing in one plane but shrinking in the other. With non-zero $\alpha$ 's at the IP it is possible to tune $\beta \max$ in the doublets - decreasing $\beta \max$ in one plane \& increasing it in the other - until an acceptable match is reached between the ratio of $\beta$-functions $\&$ the number of separators available. In addition, by tuning $\alpha^{*}$ to reduce $\beta \max$ it might also be possible to decrease $\beta^{*}$, which will tend to increase the luminosity. All of these issues need to be better understood.

\section{$\Omega$}


APPENDIX A : PARAMETERS OF THE B0 COLLIDER \& FIXED TARgET LATTICES

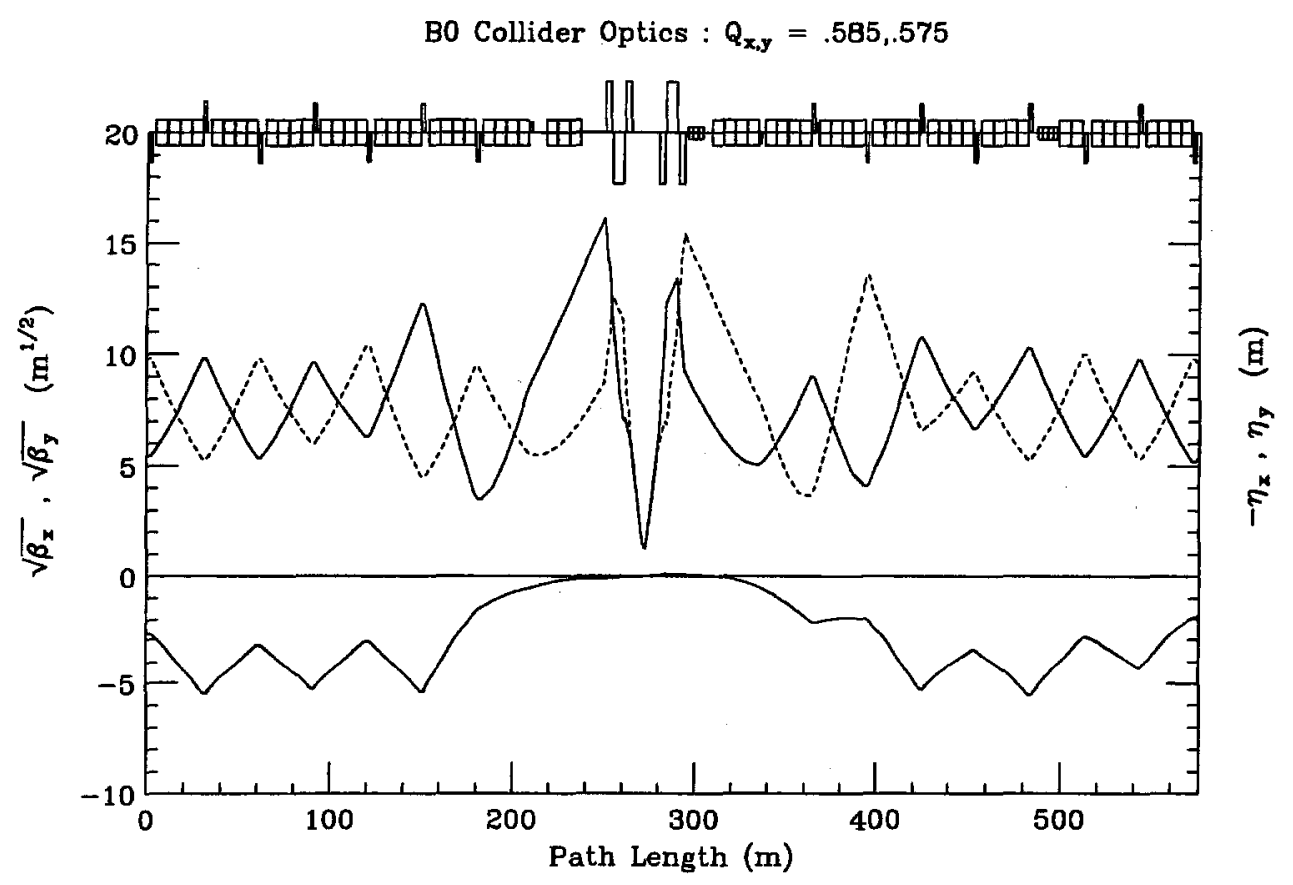

Figure A1. CDF Injection optics in Collider mode.

\begin{tabular}{|c|c|c|}
\hline Quad \# & \multicolumn{2}{|c|}{$\begin{array}{ll}\text { B0 Collider Injection Gradients } \\
\text { up } & \text { down } \\
(\mathrm{T} / \mathrm{m}) & (\mathrm{T} / \mathrm{m}) \\
\end{array}$} \\
\hline Q4 & 133.5434 & -133.5434 \\
\hline$\overline{\mathrm{Q} 3}$ & -133.4321 & 133.4321 \\
\hline$\overline{\mathrm{Q} 2}$ & 133.5434 & -133.5434 \\
\hline Q11. & 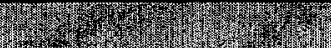 & rif \\
\hline Q5 & 26.4562 & -26.4562 \\
\hline$\overline{Q 6}$ & -26.6070 & 26.6070 \\
\hline QT6 & 8.8889 & \\
\hline QT7 & 38.0949 & -38.7098 \\
\hline QT8 & -8.6878 & 8.8835 \\
\hline QT9 & -24.8335 & 34.8228 \\
\hline QT0 & 11.7417 & -27.0644 \\
\hline QTA & $\overline{0.0}$ & \\
\hline QTB & -2.2360 & \\
\hline
\end{tabular}

Table A1. CDF IR gradients for Injection optics in Collider mode. 
Bo Fixed Target Optics : $Q_{x_{,},}=.585, .575$

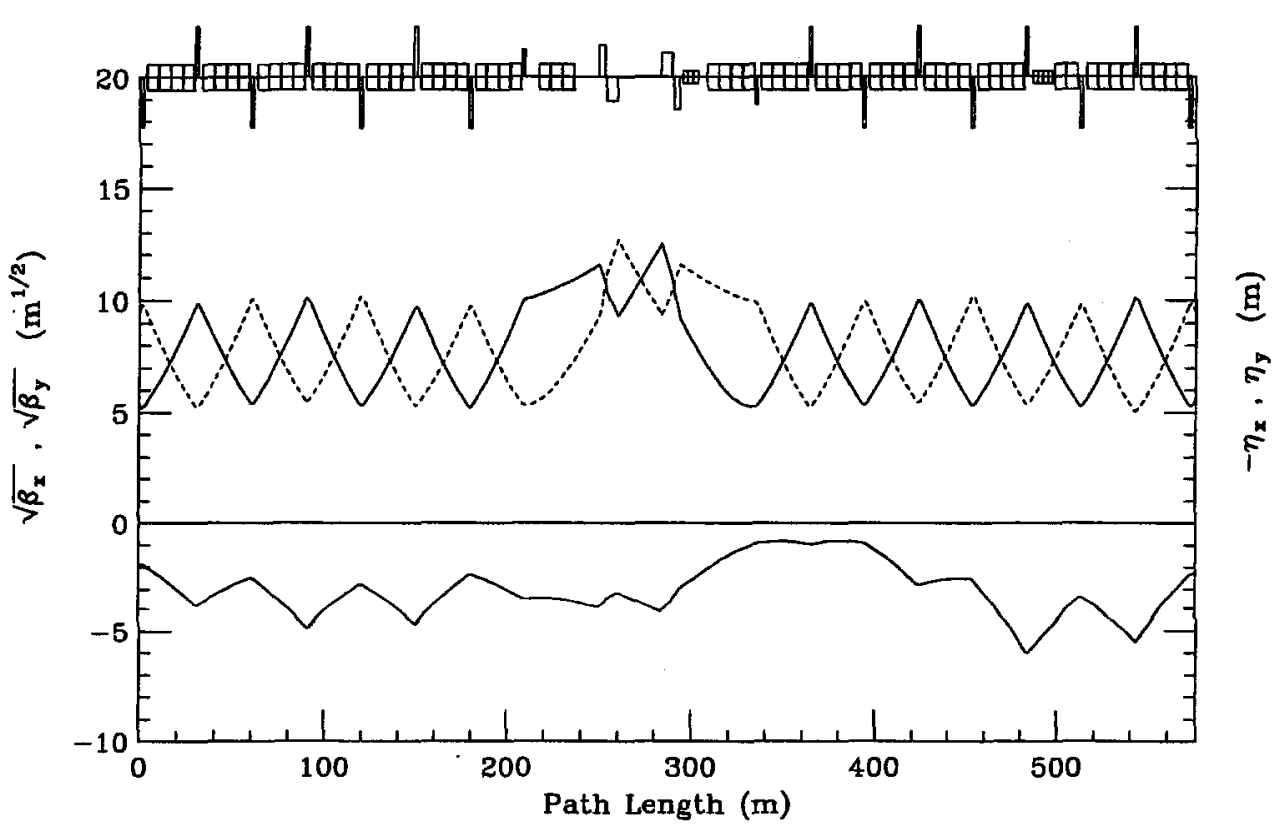

Figure A2. CDF optics in the Fixed Target lattice.

\begin{tabular}{|c|c|c|}
\hline Quad \# & \multicolumn{2}{|c|}{$\begin{array}{l}\text { B0 Fixed Target Gradients } \\
\begin{array}{ll}\text { up } & \text { down } \\
(\mathrm{T} / \mathrm{m}) & (\mathrm{T} / \mathrm{m})\end{array} \\
\end{array}$} \\
\hline Q4 & 0.0 & 0.0 \\
\hline$\overline{\mathrm{Q}}$ & -36.0874 & 36.0874 \\
\hline$\overline{\mathrm{Q} 2}$ & 48.1694 & -48.1694 \\
\hline Q & 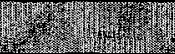 & 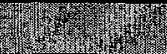 \\
\hline Q5 & 41.4443 & -41.4443 \\
\hline Q6 & 0.0 & 0.0 \\
\hline QT6 & 0.0 & 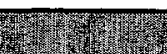 \\
\hline QT7 & 0.0 & 0.0 \\
\hline QT8 & $\overline{0.0}$ & 0.0 \\
\hline QT9 & $\overline{0.0}$ & 0.0 \\
\hline QT0 & $\overline{0.0}$ & 0.0 \\
\hline QTA & 0.0 & \\
\hline$\overline{\text { QTB }}$ & 0.0 & \\
\hline
\end{tabular}

Table A2. CDF quadrupole gradients for the Fixed Target lattice. 